\title{
Luca Morlino*
}

CNR - Opera del Vocabolario Italiano, Firenze

\section{"OVE LA SAVA NEL DANUBIO SCENDE". UNA CHIOSA SULL'ASSEDIO DI BELGRADO NELL'ORLANDO FURIOSO \\ (e in altre ottave quattro-cinquecentesche)}

\begin{abstract}
L'articolo prende in esame l'episodio dell'assedio di Belgrado nell'Orlando Furioso (canti XLIV-XLV), con particolare riferimento alle probabili allusioni a vicende storiche moderne e alla loro sovrapposizione al racconto ispirato dalle guerre tra l'Impero romano d'Oriente e i Bulgari della seconda metà dell'VIII secolo. Sulla base di un contributo di Mate Zorić trascurato negli studi ariosteschi, l'autore individua l'evento cui probabilmente allude l'Ariosto nell'assedio di Belgrado da parte dei Turchi nel 1456, integrando e approfondendo l'ipotesi dello studioso croato con alcuni nuovi dati e rilievi relativi al contesto storico-letterario.
\end{abstract}

Parole chiave: Ariosto, Orlando Furioso, assedio di Belgrado, guerre d'Oriente, letteratura e storia.

Il verso citato nel titolo circostanzia con precisione l'arrivo di Ruggiero a Belgrado nel canto XLIV dell'Orlando Furioso (79: 1), in cui il discendente di Ettore promesso sposo di Bradamante interviene in difesa dei Bulgari, proprio alla confluenza della Sava e del Danubio, contro l'esercito dell'imperatore romano d'Oriente Costantino e di suo figlio Leone, rivale in amore dello stesso Ruggiero. L'ambientazione dello scontro appare piuttosto curiosa, in quanto estranea agli scenari esotici convenzionali dell'epica cavalleresca, ma non risulta tuttavia che a essa sia stata prestata sinora molta attenzione, a dispetto dell'importanza strutturale che l'episodio, introdotto dall'Ariosto al posto dei Cinque canti soltanto nella terza e definitiva redazione del poema, riveste nell'economia generale di quest'ultimo, di cui costituisce "il preludio della fine". Le interpretazioni intorno

*morlino@ovi.cnr.it

${ }^{1}$ Così Giudicetti 2010: 53-58, nel capitolo intitolato appunto "Il preludio della fine. La funzione strutturale dell'episodio di Leone e Ruggiero". 
all'inconsueto teatro della vicenda non sono comunque certo mancate, ma appaiono lacunose nella ricostruzione del quadro contestuale, cosa del resto non infrequente nel caso dei complessi e non sempre facilmente districabili rapporti tra storia e letteratura ${ }^{2}$, e rendono pertanto opportuno un nuovo intervento sulla questione, a maggior ragione dato che il più recente, come si avrà modo di vedere, prescinde completamente dai precedenti.

Date le coincidenze onomastiche, etnografiche e militari, non sembra comunque ci siano dubbi intorno al fatto che la base storica dell'episodio sia costituita dalle guerre combattute dall'imperatore romano d'Oriente Costantino V Copronimo e dal figlio Leone IV il Cazaro contro i Bulgari nella seconda metà dell'VIII secolo, più o meno contemporanee alla cronologia delle vicende carolingie narrate nel poema e abbastanza coerenti con queste anche perché Costantino cercò, sia pure invano, di far sposare il figlio Leone con Gisella, la sorella di Carlomagno ${ }^{3}$. Eppure, la vivida rappresentazione dello scontro da parte dell'Ariosto e la sua stessa ambientazione a Belgrado, l'antica Singidunum, che invero cominciò a essere chiamata come oggi solo un secolo dopo tali vicende $(878)^{4}$, ha indotto alcuni dei non molti studiosi che hanno trattato la questione a ipotizzare che a questo strato di base si sia sovrapposto qualche altro evento più vicino nel tempo all'autore. Così, Ezio Levi ha pensato alle imprese orientali del comandante della compagnia catalana degli almogavari Ruggiero da Fiore a cavallo tra la fine del XIII e l'inizio del XIV secolo narrate qualche decennio più tardi nella Cronica di Ramon Muntaner e rievocate poi a fine Quattrocento nel Tirant lo blanch, il romanzo cavalleresco di Joanot Martorell da cui l'Ariosto trasse ispirazione per l'episodo di Ginevra nel canto IV $^{5}$. Pio Rajna è invece sceso sino alla conquista di Belgrado da parte dei Turchi di Solimano il Magnifico nel 1521 , indicando peraltro per errore al posto di questa data quella, ancor più a ridosso della terza redazione del poema Furioso, del 1529, relativa invece al primo assedio di Vienna e ripresa per inerzia anche in alcuni commenti del poema ${ }^{6}$. Al riguardo Mate Zorić ha tuttavia lucidamente obiettato che l'evento indicato da Rajna non è congruente dal punto di vista dell'esito

${ }^{2} \mathrm{Al}$ riguardo mi permetto di rimandare alle considerazioni introduttive di un mio intervento relativo al primo poema cavalleresco composto per un marchese del casato estense, la Pharsale franco-veneta composta da Niccolò da Verona nel 1343: Morlino 2014: 27-28.

${ }^{3}$ Cfr. Segre 1964: 1414 (n. 52), Zorić 1988: 10-11, Floris 2012: 187-192. Per le vicende storiche, cfr. Ostrogorsky 1968: 151-169.

${ }^{4}$ Cfr. Norris 2009: 7.

${ }^{5}$ Cfr. Levi 1933a/b, cui Segre 1964: 1414 (n. 52) rimanda “con cautela”. Per l'episodio di Ginevra, cfr. Rajna 1900²: 149-153.

${ }^{6}$ Cfr. Rajna 1900²: 601-604, Zorić 1988: 15 e n. 45. 
in rapporto all'episodio ariostesco, in cui, grazie all'apporto decisivo di Ruggiero, Belgrado viene invece alla fine liberata dall'assedio, proprio come realmente avvenuto alcuni decenni prima, nel 1456, quando l'esercito ungherese comandato da János Hunyadi (la città serba era all'epoca un avamposto magiaro), affiancato in modo decisivo dalle truppe cristiane ispirate dalla predicazione del legato apostolico Giovanni da Capestrano, riuscì a fermare l'avanzata del sultano Maometto II, già vincitore di Costantinopoli tre anni prima. Lo studioso croato ha pertanto convincentemente proposto di risalire a questa data, ricordando inoltre a ragione che, rispetto al nucleo storico principale di età carolingia, l'Ariosto si concede non poche volte nel poema la libertà "di spingere in un passato leggendario vicende più o meno recenti", determinando una "stratificazione e comprensione di tempi storici, sincronizzati al 'servizio' di messaggi attuali" una questione più generale di grande importanza per l'interpretazione del Furioso, anche in chiave contrastiva rispetto al modello boiardesco, che è stata oggetto di diversi contributi recenti e che costituisce anzi uno dei filoni di ricerca più interessanti e vivaci degli studi ariosteschi degli ultimi anni $^{8}$. Anche sulla scia di tali contributi non sembra pertanto inutile tornare sull'assedio di Belgrado nel Furioso e integrare la persuasiva interpretazione di Zorić. Ciò appare anzi tanto più necessario dato che non risulta che essa sia stata recepita, come invero merita, negli studi ariosteschi, nemmeno nell'ultimo contributo dedicato alla questione, la cui autrice, Gonaria Floris, è tornata apoditticamente al decennio indicato da Rajna, datando però l'evento cui avrebbe alluso l'Ariosto al 1526, anno per la verità della più celebre battaglia di Mohács. Senza discutere le ipotesi degli studiosi precedenti, nemmeno quella dello stesso Rajna, citato soltanto all'inizio in termini generali, e soprattutto senza circostanziare adeguatamente i fatti accaduti in tale occasione, la studiosa si è infatti limitata a scrivere in modo peraltro poco perspicuo, e senza precisi riferimenti a fonti storiografiche, che "nella famosa battaglia di Mohács, del 1526, Solimano il Magnifico riportò la vittoria contro gli Ungheresi, sconfiggendo Belgrado", ritenendo ciò sufficiente per affermare poi che "come si è messo in luce, ci fu real-

${ }^{7}$ Cfr. Zorić 1988: 12-15 e 9-10 per le citazioni. Al saggio di Zorić si rimanda anche per altra bibliografia non citata nelle note seguenti. Sulla vicenda storica, cfr. Hofer 1931: Pellegrini 2013: 292-315.

${ }^{8}$ Oltre al fondamentale Casadei 1988, cfr. in particolare Scianatico 2005 e Morato 2012, ai quali si rinvia anche per la bibliografia pregressa. Segnalo inoltre in bozze che Tina Matarrese è intervenuta al convegno "Carlo Magno in Italia e la fortuna dei libri di cavalleria" tenutosi a Zurigo (6-8 maggio 2014) con una comunicazione intitolata "La letteratura cavalleresca dal fantastico alla storia: le 'guerre d'Italia' tra cantari e poema ariostesco". 
mente nel 1526 l'assedio a Belgrado" e che quindi, ma in realtà con una mera petizione di principio, "togliendo il velo della narrazione fantastica e del Medioevo, nelle citate stanze 78-80 del XLIV canto, ecco allora che si intravede il nuovo assedio di Belgrado, del 1526"9. Quest'ultimo risale in realtà, come già indicato, al 1521, mentre cinque più anni più tardi, proprio dall'avamposto di Belgrado, ormai occupato dai Turchi, questi ebbero modo di sferrare l'attacco decisivo contro gli Ungheresi, causando la morte del loro re Luigi II Jaghellone e la fine del loro regno. Al di là dei limiti di metodo e delle imprecisioni storiche che inficiano il contributo della studiosa, anche per l'interpretazione da lei proposta vale comunque l'obiezione di fondo già avanzata da Zorić nei confronti di Rajna: "la contraddizione 'interna' al motivo centrale dell'episodio", ovvero, per l'appunto, il fatto che in quest'ultimo "è celebrata una difesa vittoriosa ed esemplare dell'importantissima città e non una resa poco gloriosa (cioè quella del 1521), anche se inevitabile davanti al dilagante pericolo turco"10.

Al di là dell'ultimo contributo citato, quello di Zorić va invece integrato in primo luogo da un altro punto di vista, in tutti i sensi più laterale, ma non meno interessante, relativo non già alla fonte storica, bensì a quella geografica relativa alle altre due località slave citate nello stesso episodio dall'Ariosto, Novengrado e Beleticche (XLV, 10: 2 e 11:3). A tal proposito Alexandre Doroszlaï ha fatto tabula rasa di tutte le interpretazioni, più o meno approssimative, avanzate in precedenza, individuando l'unica fonte che riporta entrambi i toponimi nella stessa forma (o quasi) con cui occorrono nel Furioso: si tratta della carta geografica Ungaria, prodotta probabilmente nell'atelier veneziano di Giovanni Andrea Valvassore intorno al 1520, che ha permesso allo studioso di identificare tali località rispettivamente con la città serba di Novi Pazar e con un Castellum Belethyncz a ovest del Danubio menzionato in vari documenti tra il 1332 e il 1519, ma poi, almeno a quanto pare, scomparso ${ }^{11}$. L'accurata ricostruzione di Doroszlaï - il quale ha inoltre il merito di aver ricordato che nel verso qui citato nel titolo

${ }^{9}$ Floris 2012: 192 e 196. La studiosa si limita infatti a sintetizzare molto rapidamente le tappe dell'avanzata turca nei primi decenni del Cinquecento sulla base di un rinvio generico complessivo al solo Schreiber 1986, che tuttavia non fa alcun riferimento a un assedio di Belgrado nel 1526, come nemmeno Goodwin 2009. Ciò appare tanto più anomalo a fronte tanto della maggiore dovizia di particolari e riferimenti bibliografici sulle vicende di età carolingia, quanto dell'esibizione iniziale, del tutto accessoria rispetto alla questione trattata, di auctoritates quali Baltrušaitis, Todorov e il feticcio degli studi postmoderni e postcoloniali più à la page, Said.

${ }^{10}$ Zorić 1988: 15.

${ }^{11}$ Cfr. Doroszlaï 1998: 165-180 e 217-228, cui si rimanda anche per la discussione critica della bibliografia pregressa, in cui per la verità lo studioso non fa riferimento al saggio di Zorić, che ha invece proposto di identificare le due località con la fortezza serba 
l'Ariosto aveva inizialmente scritto per errore Drava correggendosi poi in un secondo tempo e rivelandosi così più preciso anche di uno storico quale Marin Sanudo ${ }^{12}$ - ha implicitamente confermato ma soprattutto precisato un aspetto della generica supposizione avanzata da Arturo Cronia, secondo cui le conoscenze storico-geografiche alla base dell'episodio in questione deriverebbero appunto da "cronache, storie, rapsodie, cosmografie ecc. latine o italiane"13.

Questa è d'altronde la via che ha seguito anche lo stesso Zorić a proposito del racconto storico dell'assedio di Belgrado, arrivando a postulare che la fonte usufruita da Ariosto sia stata la Chronica Hungarorum dello storico e pronotario del regno ungherese János Turóczy, pubblicata nel 1488, di cui Herbert Frenzel ha documentato la presenza nella Biblioteca Estense, in due esemplari, probabilmente portati a Ferrara dall'umanista Pandolfo Collenuccio, il quale tradusse in volgare un capitolo dell'opera per il duca Ercole I d'Este ${ }^{14}$. L'incrocio di questi dati è certamente significativo e anzi indubbiamente molto suggestivo, anche se, come ha riconosciuto con la dovuta cautela lo stesso Zorić, quella da lui individuata rappresenta una fonte "possibile, ma non unica", se si considera che l'assedio di Belgrado "ebbe una larga eco italiana ed europea" 15 . Al riguardo, lo studioso si è tuttavia limitato a citare, oltre alla Chronica Hungarorum, un passo delle Istorie fiorentine di Machiavelli ${ }^{16}$, mentre per farsi un'idea più adeguata della risonanza dell'episodio in Italia, e quindi per interpretare meglio la sua più che probabile eco nel Furioso, si può ora ricorrere agli importanti contributi di Roberto Rusconi, Paolo Evangelisti, Francesco Bruni e Ioan Aurel Pop dedicati rispettivamente alla fortuna agiografica di ispirazione francescana, a livello tanto figurativo quanto letterario, di Giovanni da Capestrano, che fu, anche più di Hunyadi, la vera anima della difesa di Belgrado, e alle relazioni sull'episodio degli ambasciatori della Repubblica Serenissima di Venezia ${ }^{17}$.

di Smederevo, costruita proprio nel XV secolo, e quella bulgara di Belogračik: cfr. Zorić 1988: 17-21.

${ }^{12}$ Doroszlaï 1998: 174.

${ }^{13}$ Cronia 1958: 147.

${ }^{14}$ Zorić 1988: 15-20, con riferimento a Frenzel 1955: 161-179.

${ }^{15}$ Zorić 1988: 21 e 15.

${ }^{16}$ Ibidem.

${ }^{17}$ Cfr. Rusconi 1987, Evangelisti 1998: 231-297, Bruni 2003: 384-392, Pop 2008, ai quali si rimanda anche per le fonti citate e per la bibliografia pregressa. Riguardo a Giovanni da Capestrano, Zorić 1988: 23 (n. 66) si è limitato invece a citare la testimonianza di inizio Novecento del padre minorita Aniceto Chiappini, commentata da Di Gregorio 1986. 
Questi contributi non vengono qui richiamati, beninteso, ai fini dell'identificazione di un'altra possibile fonte dell'Ariosto: non si pretende ovviamente di dimostrare che l'autore del Furioso abbia letto la testimonianza in volgare abruzzese o piuttosto quella in latino di Giovanni da Tagliacozzo, uno dei compagni di Giovanni da Capestrano, che pure rappresenta "una sorta di incunabolo delle legendae capestranesi" e in particolare della vita di quest'ultimo scritta da Cristoforo da Varese nel 1462-1463, di cui, per sollecitare la canonizzazione del predicatore, nel 1479 venne anche pubblicato un volgarizzamento, realizzato da Teofilo da $\mathrm{Como}^{18}$. Il fine consiste piuttosto nella sottolineatura della notevole diffusione di tali leggende, dato che queste ultime costituiscono comunque nel loro complesso una base utile per provare davvero a "spiegare la scelta di Belgrado"19, che appare, sia detto per inciso, tanto più degna di nota se si considera che anche nella stessa mappa letteraria serba la città occupa curiosamente uno spazio tutto sommato relativo ${ }^{20}$.

Ciò è del resto quanto lo stesso Zorić ha giustamente ritenuto necessario, contestando la mancata presa di posizione al riguardo in particolare da parte di Levi e di Cronia, sebbene, a ben guardare, si tratti di un proposito che anch'egli ha poi realizzato soltanto in parte. Zorić, infatti, ha sì indicato una possibile fonte dell'Ariosto, ma in fondo senza giustificare la ragione poetica del presunto prelievo ariostesco dalla Chronica Hungarorum, senza cioè arrivare concretamente a "spiegare l'importanza che è attribuita dall'Ariosto all'assedio della città serba (all'epoca dell'Ariosto anche ungherese)" in quello che è uno tra gli episodi "meglio elaborati nel Furioso" e "il più cospicuo episodio slavomeridionale del Furioso"21. In altri termini, per riprendere i termini di un nodo centrale nella storia della critica tout court ma in particolare proprio di quella ariostesca, Zorić si è fermato o è comunque andato poco oltre il piano della ricerca erudita delle fonti, di cui in generale l'esempio principe e ancor oggi fondamentale è l'opera di Rajna, senza completare il quadro dell'interpretazione, dato che un singolo episodio come un'intera opera, quando si tratti di vera opera letteraria, per dirla con Benedetto Croce, "non si risolve nelle fonti" 22 . Al contrario, i

\footnotetext{
${ }^{18}$ Rusconi 1987: 29-30 e 32, cui si rimanda anche per le fonti citate.

${ }^{19}$ Zorić 1988: 13.

${ }^{20}$ Cfr. Mitrović 2007.

${ }^{21}$ Zorić 1988: 13 e 10. Per gli altri episodi minori, si veda ivi, 3-9.

${ }^{22}$ Croce 1909: 487. A questa considerazione, alla pagina successiva, Croce ne fa se-
} guire altre invero non condivisibili, in cui la critica delle fonti viene sminuita con eccesso polemico, e con implicito riferimento proprio a Rajna $1900^{2}$, criticato invero esplicitamente in precedenza (cfr. Croce 1903), a "cosa affatto oziosa, prodotto di mera e vana curiosità [...] semplice passatempo erudito", in termini non dissimili da quelli rivolti tre decenni più 
contributi citati, pur senza mai citare l'Ariosto, che fuoriesce evidentemente dal quadro della loro trattazione e dal loro intento, per l'eterogenesi dei fini consentono di comprendere meglio l'ambientazione dell'episodio di Leone e Ruggiero o in altri termini come mai quest'ultimo arrivi proprio "dentro a Belgrado" (XLIV, 80: 1).

Per rispondere alla questione occorre partire dal vero e proprio spirito di crociata che dovette caratterizzare l'assedio del 1456 e che si desume dalle relative testimonianze diplomatiche, letterarie e figurative attentamente esaminate dagli studiosi citati. Si tratta di un aspetto lasciato al contrario un po' sullo sfondo e non sottolineato in maniera adeguata da Zorić, che ha segnalato piuttosto la nuda realtà storica, ovvero che "nei secoli XV e XVI, Belgrado fu un importante caposaldo del Regno ungarico, la sua 'chiave' e la porta meridionale intorno a cui furono combattute aspre lotte" 23 , sulla scia della definizione di Marin Sanudo già citata da Rajna: "la chiave di questo regno e del tutto" 24 . Tutto vero, beninteso, e certamente doveroso da ricordare in un buon commento dell'episodio, al pari delle considerazioni relative a quest'ultimo dello stesso Rajna e a quelle più generali di Nicola Zingarelli richiamate da Zorić, secondo cui rispettivamente l'Ariosto mette qui in scena "una vera battaglia, con mosse logiche e appropriate al terreno, ch'egli si studia di rappresentarsi conformemente alla realtà, dando rilievo al fiume - la Sava - che vi scorre, e non già, come di solito accade, in modo fantastico e scolorito" 25 , dando così prova di essere tutt'altro che "indifferente alla realtà storica contemporanea" 26 , ma al contrario a conoscenza "di tutti i paesi di Europa, quanto a geografia, condizioni politiche, uomini, sovrani, non per informazione fantastica [...] ma precisa e vasta" 27 . Se pertanto è vero, secondo lo stesso Zingarelli, che "non sarebbero degni di scusa" i lettori moderni del Furioso interessati "alle fantasie dell'Ariosto solo per quello che hanno di immediato e materialmente sensibile" 28 , è però vero anche che non sarebbero degni di scusa nemmeno i lettori interessati soltanto alla realtà storica considerata stricto sensu e non estesa inoltre a quella rappresentazione della realtà che, a suo modo, finisce per costituire pur sempre parte integrante della stessa realtà, ovvero in altri termini alla considerazione dell'immaginario collettivo che di quest'ultima, nella lun-

tardi contro la "critica degli scartafacci". Per un approccio contemporaneo alla questione delle fonti del Furioso, cfr. almeno Bologna 1998: 202-206.

${ }^{23}$ Zorić 1988: 9.

${ }^{24}$ Rajna $1900^{2}: 602$.

${ }^{25}$ Ivi, 601; cfr. Zorić 1988: 14.

${ }^{26} \mathrm{Ivi}, 21$.

${ }^{27}$ Zingarelli 1934: LIX.

${ }^{28}$ Ibidem. 
ga durata è, in un caleidoscopico andirivieni, al contempo conseguenza e presupposto.

L'immaginario collettivo cui si fa qui riferimento consiste in quella vera e propria "ossessione turca" - come l'ha ben definita Giovanni Ricci concentrando lo sguardo significativamente, pars pro toto, proprio sulla Ferrara dell'Ariosto ${ }^{29}$ - che ha caratterizzato la mentalità europea nell'età moderna: si tratta di quel "pericolo turco" che più o meno direttamente influenza molta letteratura rinascimentale e anzi già la trecentesca Entrée d'Espagne franco-veneta, come hanno indicato Carlo Dionisotti e sulla scia di quest'ultimo, a ritroso, Alberto Limentani ${ }^{30}$. A tal riguardo va notato per la verità che Dionisotti accenna sì di passaggio anche all'Ariosto, ma in chiave contrastiva, come esempio del "crescente riserbo e distacco dalla contesa storico-politica della letteratura di avanguardia" rispetto alla grande fortuna, già nel Quattrocento, del motivo del "pericolo turco" nella letteratura popolare ${ }^{31}$. Eppure, ferma restando la distinzione di fondo tracciata da Dionisotti sulla base della questione della libertà italiana, prioritaria agli occhi dell'Ariosto rispetto a quella di una nuova crociata, è anche vero che, per gli intrecci che sempre legano la politica interna a quella internazionale, l'una non è comunque, né potrebbe essere, del tutto disgiunta dall'altra. Ne è prova del resto la digressione del canto XVII (73-79) rievocata dallo stesso Dionisotti, in cui l'Ariosto esorta Spagnoli, Francesi, Svizzeri e Tedeschi a combattere contro "il Turco immondo" anziché tra di loro per spartirsi l'Italia, rivolgendosi infine per questo stesso motivo anche al papa ${ }^{32}$. A ciò va aggiunto che, come ha notato ancora Dionisotti, il compito della letteratura popolare ispirata alle guerre d'Oriente "non era soltanto di dar voce a qualunque evento nuovo, ma anche e in ispecie di iterare eventi e motivi remoti, e insomma di mescolare e insieme risolvere il vecchio e il nuovo in una favola evasiva" ${ }^{33}$. Si tratta di un procedimento comune alla tradizione cavalleresca che culmina, dal punto di vista della qualità stilistica e dell'organizzazione strutturale, nello stesso Ariosto, di cui il compianto Marco Praloran ha messo in risalto la "concezione grandiosa che riesce ad assimilare il tempo, a dominarlo ed a organizzarlo in modo così 'fine' da mettere in luce contemporaneamente la progressione drammatica degli

${ }^{29}$ Cfr. Ricci 2002 e inoltre, ma certo senza alcuna pretesa di esaustività, Ricci 2008 , Formica 2012.

${ }^{30}$ Cfr. Dionisotti 1964, Limentani 1980.

${ }^{31}$ Dionisotti 1964: 211 per la citazione, con riferimento alle due pagine precedenti per la letteratura popolare.

${ }^{32}$ Per la fortuna di questo motivo, con riferimento alla pubblicistica dell'epoca, cfr. anche Casadei 1988: 54.

${ }^{33} \mathrm{Ivi}, 209$. 
eventi, logico-drammatica in senso aristotelico, e il senso che acquistano gli eventi anche sulla linea verticale, per imitationem diremmo, in senso musicale, nel confronto con altri eventi cui sono assimilabili" ${ }^{34}$.

Alla letteratura popolare delle guerre d'Oriente appartiene un altro tassello significativo da aggiungere ai contributi storico-critici richiamati in precedenza. Si tratta di un poemetto quattrocentesco di sessantadue ottave dedicato proprio all'assedio di Belgrado del 1456 e intitolato per l'appunto Istoria del gran Turcho quando fo roto a Belgrado in Ongaria, composto a ridosso degli eventi e comunque entro il 1460 secondo Anna Cornagliotti, che ne ha procurato l'edizione sulla base dell'unico testimone a quanto risulta conservato, il ms. 1602 della Biblioteca Central de Catalunya, risultante dallo smembramento di un più ampio codice di origine padovana, e significativamente di ambito francescano, conservato sotto la segnatura 5.3.24 alla Biblioteca Colombina di Siviglia ${ }^{35}$. Il poemetto, che risulta in generale piuttosto poco noto ${ }^{36}$, è degno di attenzione in primo luogo perché riguarda, come ha opportunamente notato la stessa Cornagliotti, un "momento storico, così importante nella epopea della lotta contro i Turchi nel $\mathrm{XV}$ secolo [che] non pare aver avuto, sul piano letterario, la medesima fortuna e divulgazione che ebbe invece il disastro del 1453" 37 . L'osservazione della studiosa va inoltre estesa anche agli altri "fatti bellici di più clamorosa risonanza che punteggiano l'ascesa ottomana a spese della Cristianità e segnatamente della Serenissima Repubblica", ovvero, dopo la caduta di Costantinopoli, in particolare l'eccidio di Negroponte (1470), l'assedio di Scutari (1474) e la presa di Modone (1500) $)^{38}$. Se si considera il maggior numero di testimonianze letterarie relative a questi eventi e il fatto che buona parte di esse ha raggiunto la dignità della stampa, sia pure per lo più effimera $^{39}$, sembrerebbe di poter affermare che alla minore fortuna del poemetto sulla vittoria cristiana a Belgrado abbia paradossalmente contribuito

${ }^{34}$ Praloran 2009: 313.

${ }^{35}$ Cornagliotti 1984.

${ }^{36}$ Devo la conoscenza del cantare alla citazione, dovuta a interessi puramente linguistici, da parte di Tomasin 2004: 183 e 190 (nn. 318 e 332).

${ }^{37}$ Cornagliotti 1984: 8 la quale basa tale affermazione sul corpus di lamenti storici edito da Medin - Frati 1887-1894. Una conferma in proposito proviene dal corpus di ristampe anastatiche curate da Beer - Diamanti - Ivaldi 1988-1989, vol. IV, dedicato alle guerre contro i Turchi (1453-1570); per le notizie bibliografiche si veda il vol. I, 149-211. Sull'eco della caduta di Costantinopoli, cfr. più in generale Pertusi 1976.

${ }^{38}$ Lippi 2001, 140. Su queste vicende e più in generale sui rapporti tra Venezia e i Turchi in età moderna, cfr. Preto 1975.

${ }^{39}$ Cfr. Alfano 2011: 41, il quale nota che si tratta di "edizioni strettamente legate alla contingenza degli eventi, sorta di cronache poetiche che raccontano le battaglie maggiori [...] Questo aspetto influenza anche il carattere materiale dei testi, destinati al consumo 
proprio l'esito dello scontro, quasi a conferma e ad anticipazione, fatte salve le debite differenze, di come "riuscisse più facile la poesia del compianto che non quella del trionfo", secondo quanto notato da Dionisotti riguardo a un successo militare decisamente più epocale, cui pure furono dedicati numerosi panegirici, quale quello di Lepanto (1571), legato comunque in modo inestricabile alla precedente caduta di Cipro nello stesso anno ${ }^{40}$.

Occorre ribadire che anche in questo caso, e tanto più per le ragioni appena richiamate, non si pretende certo di aver identificato la fonte specifica dell'Ariosto, che pare del resto inattingibile con precisione, dato il rapporto puramente allusivo alle vicende belgradesi del 1456 ipotizzato da Zorić, che, anche in virtù delle integrazioni storico-critiche qui apportate, appare comunque difficilmente contestabile. I numeri delle forze in campo nei due testi inoltre non coincidono, dato che nell'Istoria i Turchi sono quarantamila, tre volte tanti gli Ungheresi ${ }^{41}$, mentre nel Furioso i Greci ventimila, quattro volte tanti i Bulgari (XLIV, 81: 1 e 82: 2); né sarebbe comunque dirimente se anche così fosse, in assenza di altri riscontri significativi, per esempio in ambito rimico, in cui, a titolo di curiosità, Belgrado è associata una volta con grado e due con guado nel Furioso (XLIV, 95: 7-8 e 98: 2-4-6), mentre con la forma veneta armado nell'Istoria (21: 7-8). Significativo è invece, ma comunque non specifico, proprio il motivo del guado, certo topico nella narrativa cavalleresca ${ }^{42}$ e inoltre qui favorito, se non quasi obbligato per l'appunto da ragioni di rima; ciò nondimeno esso lascia pensare a un'effettiva conoscenza da parte dell'Ariosto di qualche narrazione dell'assedio di Belgrado, in particolare per quanto riguarda la necessità, per dirla con le parole dell'Istoria relative al sultano e ai suoi baroni, "de saper tenire el modo o per che via | pasar podesse el fiume tute le zente soe" (15: 4-5). Fatta arrivare via nave la sua armata, il sultano ordina poi a quest'ultima di costruire un ponte:

immediato. Il collegamento con la realtà storica è confermato dal fatto che i picchi editoriali cadono a ridosso degli eventi principali”.

${ }^{40}$ Dionisotti 1964: 221. Per le celebrazioni letterarie di Lepanto, cfr. Casadei 2011, anche per la bibliografia pregressa.

${ }^{41}$ A dire il vero, per ragioni numeriche e di contiguità testuale quest'ultima precisazione (10: 6) fa forse riferimento a un'altra cifra riportata nel poemetto: "Partendosse el gran Turcho da lutani paexe | menò siego uno conto de una zente bella: | tre xento milia el mio parlar sì dixe" (7: 1-3); nel prosieguo però la cifra è più volte quella indicata: "quaranta milia si era tuta la brigata, e messe el suo vassallo atorno e atorno | e tuta quela perfida e renegata" (21: 4-6); "La zente pagana tuta molto ben in ponto | atorno de lo castelo voleno batagiare [...] Quaranta milia certo e più ne fazo conto!" (37: 1-2 e 5), "Quaranta milia si era la canagia" (44: 6).

${ }^{42}$ Cfr. Barbieri 2009. 
E quela zente niente più tardoe, subitamente intexe del Turco lo tenore; setanta due fuste l'una e l'altra se acostoe, sopra ge fezeno uno ponte de gran valore (19: 1-4).

Questi versi meritano di essere accostati, sia pure a grandi linee e senza la pretesa di istituire un rapporto diretto, a quelli del Furioso in cui i due eserciti vengono a trovarsi sulle opposte rive della Sava:

Sul fiume il Greco per gittare il ponte,

il Bulgar per vietarlo armato stava, quando Ruggier vi giunse; e zuffa grande attaccata trovò fra le due bande (XLIV, 80: 5-8).

Di qui si può procedere inoltre ai passi analoghi dell'Angelica innamorata di Vincenzo Brusantini (1550) relativi alla stessa Belgrado (anche qui, non a caso, in rima soprattutto con guado $)^{43}$, ad altre città dell'Europa sud-orientale cadute in mano turca nel Cinquecento, tra cui Buda e la già citata Mohács, e quindi anche ai principali fiumi della regione, Danubio, Sava, Drina: come ha sottolineato Rosanna Alhaique Pettinelli, la guerra combattuta in questi luoghi infatti "pone ai comandanti la necessità di adattare i propri piani alla natura del terreno, che richiede spesso di costruire ponti che consentano agli eserciti di passare quei fiumi cui si è fatto cenno" 44 . Al riguardo la studiosa ha opportunamente registrato la congruenza tra la descrizione di tali luoghi nel poema del Brusantini e quella di altri scritti dell'epoca, in particolare i Commentarii e i tre Libri delle cose de' Turchi rispettivamente di Paolo Giovio e di Benedetto Ramberti, ma stranamente senza richiamare l'episodio belgradese del Furioso che pure evidentemente costituisce il modello primario di quelli dell'Angelica innamorata, che in generale ambisce a proporsi come continuazione del capolavoro ariostesco ${ }^{45}$. Risulta quindi qui opportuno questo ulteriore incrocio di dati, a maggior ragione perché il commento di Alhaique Pettinelli relativo a questi episodi dell'Angelica innamorata si può in parte mutuare à rebours a proposito di quello dello stesso Furioso, in particolare per il richiamo, tra le "opere

${ }^{43}$ XIX, 81: 1-3 e 85: 1-5, XXXII, 17: 2-6.

${ }^{44}$ Alhaique Pettinelli 2004: 143.

${ }^{45}$ A titolo d'esempio, basti qui riportare la prima menzione della città serba nell' $A n$ gelica innamorata: "E dietro a l'Istro sopra l'alta riva | A man destra cavalca, e il cammin prende, | Passa Belgrado, ed alla Sava arriva, | Dove la Quieta nel Danubio scende" (IV, 8: 1-4); oltre al verso qui citato nel titolo, Brusantini riprende infatti anche il distico immediatamente precedente del Furioso: "E lungo l'Istro per la destra riva | tanto cavalca, ch'a Belgrado arriva” (XLIV, 78: 7-8). 
che narrano l'espansione ottomana in Europa', anche ai poemetti in ottava rima e inoltre per la precisazione relativa alla maggiore accuratezza delle descrizioni del Brusantini rispetto a questi ultimi ${ }^{46}$.

Si tratta di una conferma per altra via del parallelo proposto sinora, ancor più significativa per la considerazione d'insieme di una tradizione poetica di tipo evidentemente seriale, di cui qui è stato opportuno segnalare un singolo esempio, sia perché relativo proprio al fatto storico cui Zorić ha fatto risalire l'allusione ariostesca, sia perché assente dal corpus di riferimento di questo genere testuale e in quanto tale meritevole di maggiore attenzione, anche a prescindere dall'interesse in rapporto al Furioso ${ }^{47}$. La considerazione d'insieme di Alhaique Pettinelli è utile e importante anche perché comprensiva degli analoghi poemetti relativi alle guerre d'Italia, più volte messi in rapporto alle allusioni alla storia contemporanea del Furio$s O^{48}$, che notoriamente annovera tra le sue fonti anche opere "spesso ben poco note" e "di secondo piano" 49 . Appare pertanto verosimile che l'Ariosto abbia potuto fare ricorso a questa tradizione anche riguardo all'assedio di Belgrado, a conferma dell'assunto generale di Caretti secondo cui "la materia del Furioso preesiste quasi tutta intera all'invenzione ariostesca" ${ }^{50}$ : beninteso, alla tradizione e non necessariamente al testo che a quest'ultima è stato qui riguadagnato.

Tale rilievo è suggerito proprio dalle caratteristiche particolari di questa tradizione, che spesso attesta diverse redazioni del racconto di uno stesso episodio, alcune delle quali si può plausibilmente pensare siano anche andate perdute, proprio in ragione della labilità di questi testi, legati a fatti contingenti e soggetti a una trasmissione popolare che si può supporre in parte anche orale ${ }^{51}$. Ciò non implica tuttavia che degli eventi più lontani nel giro dei decenni si sia persa rapidamente e completamente notizia, come prova per esempio il caso del Balzino del poeta salentino Rogeri de Pacienza (1497), che nel quinto libro (vv. 633-644) riporta addirittura un breve estratto di un canto popolare serbo, in lingua originale, relativo a un altro scontro dello stesso Hunyadi contro i Turchi, avvenuto a Smederevo

${ }^{46}$ Ivi, 143-145.

${ }^{47}$ Rimane infatti da approfondire, e mi ripropongo di farlo in altra sede, il legame del poemetto con l'ambiente francescano padovano di origine della parte principale del codice conservata a Siviglia, sulla scorta delle precisazioni di Zen Benetti 1986.

${ }^{48}$ Cfr. supra, la nota 8.

${ }^{49}$ Segre 1955: 48.

${ }^{50}$ Caretti 1973: 28. Diversamente, pur con le cautele del caso, Rajna 1900²: 603 aveva riscontrato nell'episodio belgradese del Furioso "una delle parti più originali del poema".

${ }^{51}$ Cfr. supra, la nota 39. 
mezzo secolo prima (1448), ma caratterizzato dall'esito opposto rispetto a quello di Belgrado ${ }^{52}$. Analogamente, sembra lecito ritenere che proprio la serialità di questa tradizione, nei corsi e ricorsi storici, battaglia dopo battaglia, abbia potuto favorire il perpetuarsi della memoria degli eventi. Fermo restando il rilievo di Zorić riguardo all'ipotesi di Rajna richiamato all'inizio, senza il quale l'analisi rimarrebbe schiacciata sulle vicende temporalmente più vicine alla redazione definitiva del Furioso, si può anzi postulare che proprio la caduta di un avamposto strategico come Belgrado nel 1521, e tanto più per la conseguente disfatta cristiana di Mohács, abbia richiamato $e$ contrario alla memoria dei contemporanei la strenua e vittoriosa resistenza di alcuni decenni prima, a riprova della considerazione di Praloran citata in precedenza e di quanto notato inoltre da Nicola Morato: "le battaglie rappresentate nel Furioso e quelle reali, che travolgono l'Italia e l'Europa, evocate nelle parti autoriali o alluse nella diegesi stanno nel poema come elementi distanti, con funzioni distinte; non sempre conciliati ma anche in un rapporto di segreta rispondenza, consonante o dissonante"53.

Il prospettivismo temporale appena indicato e la necessaria considerazione d'insieme della tradizione dei poemetti sulle guerre d'Oriente permettono pertanto, tirando le somme di quanto detto sin qui, di completare la risposta all'interrogativo sollevato da Zorić, ovvero: perché Belgrado? Perché proprio "Ove la Sava nel Danubio scende" si è registrata la più significativa vittoria cristiana contro i Turchi prima di Lepanto, che, al di là di quanto non appaia dai repertori dei poemetti italiani sulle guerre d'Oriente, si è impressa nell'immaginario collettivo in modo maggiore di quelle, pur meno lontane nel tempo rispetto alla composizione del Furioso, riportate dal fronte cristiano in Caramania (1477), a Rodi (1480) o nello stesso golfo di Lepanto (1499), come prova tra l'altro il fatto che a essa venne dedicato anche un romance medio-inglese, il Capystranus ${ }^{54}$.

\section{BIBLIOGRAFIA}

Alfano, G. (2011). Una forma per tutti gli usi: l'ottava rima. In S. Luzzatto e G. Pedullà (a cura di), Atlante della letteratura italana, vol. II, E. Irace (a cura di), Dalla Controriforma alla Restaurazione (pp. 31-57). Torino: Einaudi.

52 Cfr. Marti 1977: 163, Pantic 1990, Guillou 2000. All'inserto fa riferimento, con altra bibliografia, anche Zorić 1988: 2 e n. 3 .

${ }_{53}$ Morato 2012: 305.

${ }^{54}$ Cfr. Shepherd 1995: 391-408. Per i poemetti italiani relativi gli altri episodi bellici citati, cfr. Beer - Diamanti - Ivaldi 1988-1989, vol. I, 164-165 e 172. 
Alhaique Pettinelli, R. (2004). Forme e percorsi dei romanzi di cavalleria. Roma: Bulzoni.

Barbieri, A. (2009). Combattere al guado: realtà storica e radici antropologiche di un motivo letterario. L'immagine riflessa, XVIII, 23-55.

Beer, M., Diamanti, D., Ivaldi, C., (a cura di) (1988-1989). Guerre in ottava rima. 4 voll. Modena: Panini.

Bologna, C. (1998). La macchina del Furioso. Lettura dell'Orlando e delle Satire. Torino: Einaudi.

Bruni, F. (2003). La città divisa. Le parti e il bene comune da Dante a Guicciardini. Bologna: Il Mulino.

Brusantini (1550). L'Angelica innamorata di Vincenzo Brusantini. (1838). Venezia: Antonelli.

Caretti, L. (1973). Ariosto e Tasso. Torino: Einaudi.

Casadei, A. (1988). La strategia delle varianti. Le correzioni storiche al terzo Furioso. Lucca: Pacini Fazzi.

Casadei, A. (2011). Panegirici per la vittoria. In S. Luzzatto e G. Pedullà (a cura di), Atlante della letteratura italana, vol. II, E. Irace (a cura di), Dalla Controriforma alla Restaurazione (pp. 224-231).

Cornagliotti, A. (1984). "Istoria del gran turcho quando fo roto a Belgrado in Ongaria": un cantare quattrocentesco di ambiente padovano. Atti dell'Istituto Veneto di Scienze Lettere ed Arti, CXLII, 7-38.

Croce, B. (1903). Storia della Critica e storia delle Critiche particolari. In B. Croce (1949). Problemi di estetica e contributi alla storia dell'estetica italiana (pp. 439-444). Bari: Laterza.

Croce, B. (1909). La ricerca delle fonti. B. Croce (1949). Problemi di estetica e contributi alla storia dell'estetica italianal (pp. 487-502). Bari: Laterza.

Croce, B. (1949). Problemi di estetica e contributi alla storia dell'estetica italianal. Bari: Laterza.

Cronia, A. (1958). La conoscenza del mondo slavo in Italia. Bilancio storico-bibliografico di un millennio. Padova: Stediv.

Di Gregorio, F. (1986). P. Aniceto Chiappini poeta: l'ode saffica "S. Giovanni da Capestrano a Belgrado". Critica letteraria, XIV, 511-518.

Dionisotti, C. (1964). La guerra d'Oriente nella letteratura veneziana del Cinquecento. In C. Dionisotti (1967). Geografia e storia della letteratura italiana (pp. 201-226). Torino: Einaudi.

Doroszlaï, A. (1988). Ptolémée et l'hippogriffe: la géographie de l'Arioste soumise à l'épreuve des cartes. Alessandria: Edizioni dell'Orso.

Evangelisti, P. (1998). Fidenzio da Padova e la letteratura crociato-missionaria minoritica. Strategie e modelli francescani per il dominio (XIII-XV sec.). Bologna: Il Mulino. 
Floris, G. (2012). L'Oriente nel Furioso tra Carlo Magno e Carlo V. Un caso di anamorfosi. In A. Cannas, T. Cossu e M. Giuman (a cura di), Xenoi: immagine e parola tra razzismi antichi e moderni (pp. 183-198). Napoli: Liguori.

Formica, M. (2012). Lo specchio turco. Immagini dell'Altro e riflessi del Sé nella cultura italiana d'età moderna. Roma: Donzelli.

Frenzel, H. (1955). Von der Olimpia-Episode zu den Parerga des "Orlando Furioso". Germanisch-Romanische Monatsschrift, XXXVI, 161-179.

Giudicetti, G. (2010). Mandricardo e la melanconia. Discorsi diretti e sproloqui nell'Orlando Furioso. Bruxelles: Peter Lang.

Goodwin, J. (2009). I signori degli orizzonti. Una storia dell'impero ottomano. Torino: Einaudi.

Guillou, A. (2000). Une fête slave à Gioia del Colle (1 $1^{\mathrm{er}}$ juin 1497). In Studi in onore di Giosuè Musca (pp. 221-223). Bari: Dedalo.

Hofer, J. (1931). Der Sieger von Belgrad 1456. Historisches Jahrbuch, LI, $163-212$.

Levi, E. (1933a). L'"Orlando Furioso" come epopea nuziale. Archivum romanicum, XVII, 459-496.

Levi, E (1933b). L'epopea di Ruggero. In G. Fusai (a cura di), Lodovico Ariosto poeta e commissario in Garfagnana, nel quarto centenario dalla morte (pp. 139-162). Arezzo: Zelli.

Limentani, A. (1980). Venezia e il "pericolo turco" nell" "Entrée d'Espagne". In A. Limentani (1992). L' "Entrée d'Espagne" e i signori d'Italia (a cura di M. Infurna e F. Zambon; pp. 358-378). Padova: Antenore.

Lippi, E. (2001). 1517: l'ottava al servizio del Sultano. In E. Lippi (2003). Contributi di filologia veneta (pp. 139-188). Treviso: Antilia.

Marti, M. (1977) (a cura di). Rogeri de Pacienza, Opere. Lecce: Milella.

Medin, A. e Frati, L. (1887-1894) (raccolti e ordinati a cura di). Lamenti storici dei secc. XIV, XV e XVI, 3 voll. Bologna: Romagnoli.

Mitrović, M. (2007). Belgrado nella mappa letteraria serba. In M. Böhmig e A. D'Amelia (a cura di), Le capitali nei paesi dell'Europa centrale e orientale (pp. 159-176). Napoli: D'Auria.

Morato, N. (2012). Meliadus, Rodomonte, il mostro. Guerra e assalto nel primo "Furioso". L'Immagine Riflessa, XXI, 287-308.

Morlino, L. (2013). Echi e riflessi storico-politici nella letteratura francoveneta: il caso della "Pharsale" di Niccolò da Verona. In Z. Murat e S. Zonno (a cura di), Medioevo Veneto e Medioevo Europeo: identità e alterità (pp. 27-38). Padova: Padova University Press.

Norris, D. A. (2009). Belgrade. A cultural History. Oxford: Oxford University Press. 
Ostrogorsky, G. (1968). Storia dell'impero bizantino. Torino: Einaudi.

Pantić, M. (1990). Un ignoto canto popolare del XV secolo. Italica Belgradensia, III, 47-66.

Pellegrini, M. (2013). Le crociate dopo le crociate. Da Nicopoli a Belgrado (1396-1456). Bologna: Il Mulino.

Pertusi, A. (1976) (testi a cura di). La caduta di Costantinopoli. Milano: Mondadori - Fondazione Lorenzo Valla.

Pop, I. A. (2008). La battaglia di Belgrado (1456) nelle testimonianze venete. In C. Maltezou, P. Schreiner e M. Losacco (a cura di), Philanagnostes. Studi in onore di Marino Zorzi (pp. 339-348). Venezia: Istituto ellenico di Studi bizantini e postbizantini.

Praloran, M. (2009). La tradizione cavalleresca in Italia e "Mimesis". In I. Paccagnella e E. Grigori (a cura di), L'eredità di Auerbach (pp. 309-323). Padova: Esedra.

Preto, P. (1975). Venezia e i Turchi. Sansoni: Firenze.

Rajna, P. $\left(1900^{2}\right)$. Le fonti dell'Orlando furioso, ristampa della seconda edizione 1900 accresciuta d'inediti (a cura e con presentazione di F. Mazzoni, 1975, pp. 601-604). Firenze: Sansoni.

Ricci, G. (2002). Ossessione turca: in una retrovia cristiana dell'Europa moderna. Bologna: Il Mulino.

Ricci, G. (2008). I Turchi alle porte. Bologna: Il Mulino.

Rusconi, R. (1987). Giovanni da Capestrano: iconografia di un predicatore nell'Europa del '400. In Predicazione francescana e società veneta nel Quattrocento: committenza, ascolto, ricezione (pp. 25-53). Padova: Centro Studi Antoniani.

Schreiber, G. (1986). I Turchi. Sulle tracce di un grande impero. Milano: SugarCo.

Scianatico, G. (2005). Le "moderne cose". Storia contemporanea nel "Furioso". In T. Matarrese e C. Montagnani (a cura di), Il principe e la storia. Atti del Convegno, Scandiano, 18-20 settembre 2003 (pp. 223-238). Novara: Interlinea.

Segre, C. (1955). La biblioteca dell'Ariosto. In C. Segre (1966). Esperienze ariostesche (pp. 45-50). Pisa: Nistri-Lischi.

Segre, C. (1964) (a cura di). Ludovico Ariosto, Orlando Furioso. Milano: Mondadori.

Shepherd, S. H. A. (1995) (a cura di). Middle English Romances. New York: Norton.

Tomasin, L. (2004). Testi padovani del Trecento. Padova: Esedra.

Zen Benetti, F. (1986). Scheda di Cornagliotti 1984. Quaderni per la Storia dell'Università di Padova, XIX, 215. 
Zingarelli, N. (1934) (a cura di). Orlando Furioso di L. Ariosto. Milano: Hoepli.

Zorić, M. (1988). L'Ariosto, gli Schiavoni e l'assedio di Belgrado. In M. Zorić (1989), Italia e Slavia. Contributi sulle relazioni letterarie italojugoslave dall'Ariosto al D'Annunzio (pp. 1-24). Padova: Antenore.

\author{
“OVE LA SAVA NEL DANUBIO SCENDE”. A NOTE ON THE SIEGE \\ OF BELGRADE IN ARIOSTO'S ORLANDO FURIOSO \\ (AND IN OTHER POEMS 'IN OTTAVA RIMA' \\ OF XV AND XVI CENTURY)
}

\begin{abstract}
Summary
The article focuses on the episode of the siege of Belgrade in Ariosto's Orlando Furioso (cantos XLIV-XLV), with particular reference to the probable allusions to contemporary historical events and their overlap to the tale inspired by the wars between the Byzantine Empire and the Bulgarians in the second half of the eighth century. On the basis of an article of Mate Zorić neglected in Ariosto studies, the author identifies the event to which Ariosto probably alludes in the siege of Belgrade by the Turks in 1456, completing the hypothesis of the Croatian scholar with a deeper analysis of the historical and literally context.

Keywords: Ariosto, Orlando Furioso, siege of Belgrade, Orient's wars, literature and history.
\end{abstract}


